\title{
Take-away milk tea intake was associated with cholesterol independent of adiposity in young adults
}

\author{
$\underline{\text { Xin Liu }}^{1}$, Huan Chang ${ }^{2}$, Mengnan $\mathrm{Lu}^{3}$, Huangtao $\mathrm{Chen}^{3}$, Junxiang Wei ${ }^{1}$ and Xia Liao ${ }^{4}$ \\ ${ }^{1}$ Department of Epidemiology and Biostatistics, School of Public Health, Xi'an Jiaotong University Health Science \\ Center, Xi'an, China, \\ ${ }^{2}$ Department of Nutrition and Food Safety, School of Public Health, Xi'an Jiaotong University Health Science Center, \\ Xi'an, China, \\ ${ }^{3} X i$ 'an Jiaotong University Health Science Center, Xi'an, China and \\ ${ }^{4}$ Department of Nutrition, The First Affiliated Hospital, Xi'an Jiaotong University Health Science Center, Xi'an, China
}

Introduction: Take-away milk tea (TAMT) is popular among young generation, and the numbers of retails of TAMT have increased dramatically in recent years in many cities in China. Non-dairy cream is one of the major ingredients of TAMT. Concerns have been raised whether trans-fat originated from non-dairy cream may have an influence on cardio-metabolic traits. We evaluated the associations between daily intake of TAMT with plasma lipid profiles among young Chinese adults, who are the major customers of TAMT retailers.

Materials and Methods: The study population was from the phase 1 sample (104 adults) of the Carbohydrate Alternatives and Metabolic Phenotypes study. Those lacking blood samples or with a body mass index less than $18.5 \mathrm{~kg} / \mathrm{m}^{2}$ were excluded, therefore, a total of 88 subjects with an average age of 22.8 years were included in the analysis. A food frequency questionnaire with 27 items was used to collect the dietary intake. Generalized linear regression was used to evaluate the associations between TAMT intake and cholesterol levels.

Results: The estimated mean ( \pm SE) of TAMT intake was $14.4 \pm 3.4 \mathrm{ml} /$ day, with apparent differences between males $(8.8 \pm 2.7 \mathrm{ml} /$ day) and females $(17.7 \pm 5.1 \mathrm{ml} /$ day). The mean of total cholesterol of the participants was $4.1 \pm 0.1 \mathrm{mmol} / \mathrm{L}$. After adjusted for age, sex, education attainment, smoking status, alcohol drink habit, and physical activity level, daily TAMT intake was positively associated with total cholesterol (beta $\pm \mathrm{SE}=0.0053 \pm 0.0020, \mathrm{P}=0.011$ ). The association was not substantially changed with further adjustment of body fat percentage (beta $\pm \mathrm{SE}=0.0053 \pm 0.0020, \mathrm{P}=0.010$ ). Similar associations were observed for high/low density lipoprotein cholesterols. When analysis was performed by sex, the association was only observed among females (beta \pm SE $=0.0049$ $\pm 0.0022, \mathrm{P}=0.031$ ), but not in males (beta $\pm \mathrm{SE}=0.0022 \pm 0.0060, \mathrm{P}=0.703$ ).

Conclusion: In young adult Chinese, we observed an association between TAMT intake with plasma cholesterol level, independent of body adiposity.

\section{Conflict of Interest}

There is no conflict of interest 